\title{
R. Hüßtege/H. - P. Mansel (Hrsg.), BGB: Rom-Verordnungen, EuGüVo, EuPartVo, HUP, EuErbVO, Band 6
}

\author{
Alfonso-Luis Calvo Caravaca \\ Catedrático de Derecho Internacional Privado \\ Universidad Carlos III de Madrid \\ ORCID ID: 0000-0003-2236-4641
}

DOI: https://doi.org/10.20318/cdt.2019.5024

Es gibt zwei Arten von juristischen Büchern: solche, die zur Lösung von Problemen dienen, und solche, die unbrauchbar sind. Unter den ersten nehmen die deutschen Kommentare einen herausragenden Platz ein. Ein Kommentar ist eine umfangreiche und umfassende Arbeit, die, in der Regel, von mehreren Juristen mit einem unterschiedlichen beruflichen Hintergrund unter der Leitung renommierter Juristen nach der Systematik des zu analysierenden Rechtstextes ausgeführt wird. Die jetzt kommentierte Arbeit wurde von einem Team von 23 Juristen ausgeführt, darunter Universitätsprofessoren, Notare, Anwälte und ein Richter. Die Führung übernahmen R. HÜßTEGE (Vorsitzender Richter am Oberlandesgericht a.D., München) und H.-P. MANSEL (Professor für Bürgerliches Recht, Internationales Privatrecht, Rechtsvergleichung und Zivilverfahrensrecht an der Universität Köln).

Es stellt sich die Frage, aus welchen Gründen ein ausländischer Jurist diese Arbeit konsultieren sollte. Dafür gibt es mehrere gute Gründe:

a) Der Kommentar behandelt einem sehr wichtigen Thema: das Internationalen Privatrecht der Europäischen Union und insbesondere folgende internationale Instrumente: Rom-Verordnungen (Rom I [Vertragliche Schuldverhältnisse], Rom II [Außervertragliche Schuldverhältnisse], Rom III [Ehescheidung, Trennung]), EuGüVO (Eheliches Güterrecht), EuPartVO (lebenspartnerschaftliches Güterrecht), HUP (Haager Unterhaltsprotokoll, EuErbVO (Erbrecht). Die EU verfügt über ihr eigenes "System des internationalen Privatrechts", sowie das internationale Privatrecht, über das jeder Staat verfügt. Es ist wahr, dass es sich noch in der Konstruktionsphase befindet und daher unvollständig ist (H.J. SONNENBERGER, "Grenzen der Verweisung durch europäisches internationales Privatrecht", IPRax, 31, 4, 2011, 325-335). In diesem Sinne, die Kodifizierung des internationalen Privatrechts ist mehr ein Prozess als eine Tatsache (E.-M. KIENINGER, "Die weitere Kodifikation des europäischen IPR", IPRax, 37, 2, 2017, 200-208). Aufgrund seines unaufhaltsamen Wachstums und seiner Berufung zur weltweiten Regulierung des internationalen Privatrechts können wir jedoch bestätigen, dass es bereits ein echtes System des "europäischen internationalen Privatrechts" gibt, das bereits das zentrale System des internationalen Privatrechts aller Mitglieder Staaten ist (M. REQUEJO, "Reflections on the Preambles to the EU Private International Law Regulations", in Europa als Rechts- und Lebensraum: Liber amicorum für Christian Kohler zum 75. Geburtstag am 18. Juni 2018, Bielefeld, Gieseking Verlag, 2018, S. 425-439).

*3a ed., Baden-Baden, Nomos Verlagsgesellschaft, DeutscherAnwaltVerein, 2019, XLIII-1527 p. ISBN 978-3-8487-4587-6. 
b) Der Kommentar ist traditionell ein gut gebautes und gut eingerichtetes Gebäude. In der Tat kann der Leser eine strenge und sorgfältige Analyse jedes Rechtstextes finden, die von ausgewählten bibliographischen Referenzen und geeigneten juristischen Angaben begleitet wird. Ich freue mich sehr, dass eines der von diesen deutschen Juristen verwendeten Journale unsere Zeitschrift Cuadernos de Derecho Transnacional CDT (www.uc3m.es/cdt) ist, die führende spanische Fachzeitschrift für internationales Privatrecht, ausländisches Privatrecht, einheitliches Recht und internationales Wirtschaftsrecht.

c) Dieser Kommentar ist ein sehr vollständiges Werk: Es ist ein Werk, das der Analyse klassischer Themen oder den Themen, die das internationale Privatrecht des 21. Jahrhunderts kennzeichnen, nicht entgeht. Beispiel 1: Mit den entsprechenden Nuancen stellt die neue Lex Mercatoria eine Reihe von normativen Elementen dar, die von großem Nutzen sind, um die Kontroversen des internationalen Handels zwischen Unternehmen von grenzüberschreitender Dimension zu lösen (vid., ad ex., A.-L. CALVO CARAVACA/ J. CARRASCOSA GONZÁLEZ [DIR.], "Lex mercatoria y arbitraje privado internacional", in A.-L. CALVO CARAVACA/I. TIRADO MARTÍ [DIR.], UNIDROIT y la codificación internacional del Derecho privado, Valencia, Tirant Lo Blanch, 2019, im Druck). NK-BGB/Leible, Rn. 3137, setzt sich mit diesen Fragen klar und akribisch auseinander. Beispiel 2: Seit Jahren wird diskutiert, ob die Schiedsrichter in einem internationalen Schiedsverfahren mit Sitz in einem Staat der Europäischen Union Rom I-VO anwenden können oder sollten. Die Antwort hängt davon ab, wie der Ausschluss eines Schiedsverfahrens vom Artikel 1 II lit. e Rom I-VO interpretiert wird, die Möglichkeit, dass die Schiedsrichter ihre eigenen Regeln des Internationalen Privatrechts formulieren und schließlich, wenn es ein Lex fori der Schiedsgerichte gibt (vid., ad ex., v. BAR/MANKOWSKI, Internationales Privatrecht, Band II: Besonderer Teil, 2. Auflage, München, Verlag C.H. Beck, 2019, S. 255-260). Diese Diskussion spiegelt sich in NK-BGB/Brand, Das anwaltliche Mandat im internationalen Schuldrecht, Rn. 19 und 28; NK-BGB/Leible Artikel 1 ROM I, Rn. 60-61a. Beispiel 3: Das Internet hat unsere Sicht auf die Gesellschaft und die Welt, in der wir leben, verändert (vid., ad ex., A.-L. CALVO CARAVACA/J. CARRASCOSA GONZÁLEZ, Conflictos de leyes y conflictos de jurisdicción en Internet, Madrid, Editorial Colex, 2001). Es hat uns gezwungen, unter anderem die Frage des für Internetverträge, Domainregistrierungsverträge, Internetauktionen, Providerverträge, Verbraucherverträge, Internetdelikte und Produkthaftung geltende Recht zu lösen. Der Leser dieses Kommentars findet die Lösung für solche Probleme in NK-BGB/Leible, Artikel 4 ROM I, Rn. 51, 75, 111-117; NK-BGB/Lehmann, Artikel 4 ROM II, Rn. 68 und 111; NKBGB/Lehmann, Artikel 5 ROM II, Rn. 77.

d) Ein Kommentar ist ein vielseitiger Text. Es kann für die Ausbildung von Doktoranden, für den Beginn einer akademischen Forschungsarbeit und insbesondere für die berufliche Praxis der verschiedenen Rechtsberufe (Rechtsanwälte, Notare, Richter ...) verwendet werden.

Kurz gesagt, es ist ein Meisterwerk, das in keiner guten Bibliothek, die auf europäisches internationales Privatrecht spezialisiert ist, fehlen darf. Herzlichen Glückwunsch an die Direktoren und Autoren für diese ausgezeichnete Arbeit und an den Nomos-Verlag und Deutscher Anwalt Verein für diese erfolgreiche Initiative. 